\title{
Impulsive Noise Removal by Adaptive Mathematical Morphology
}

\author{
Marisol Mares Javier ${ }^{1}$, Carlos Guillén Galván ${ }^{1}$, Rafael Lemuz López ${ }^{2}$ \\ ${ }^{1}$ Benemérita Universidad Autónoma de Puebla, Facultad de Ciencias Físico \\ Matemáticas, Puebla, México \\ losyram1990@gmail.com, cguillen@f cfm. buap.mx \\ 2 Benemérita Universidad Autónoma de Puebla, Facultad de Ciencias de la \\ Computación, Puebla, México \\ rlemuz@cs. buap.mx
}

\begin{abstract}
In this work, an approach to the problem of impulsive noise removal in gray scale images is presented. We describe two models of adaptive mathematical morphology that improve the classical mathematical morphology in the removal of impulsive noise. The proposed method adjust the structuring elements to the local context of the image. The comparison with classical mathematical morphology denoising technique shows that the new approach better removes the salt and pepper noise, while preserves image details.
\end{abstract}

Keywords: Morphological Filtering, Opening, Closing, Composition, Structural Element.

\section{Introduction}

Impulsive noise (or salt and pepper), is one of the types of noise more often present in digital images. This kind of noise is caused by several factors such as, faulty memory location in hardware, problems in scanning, video sensor problems, decoding errors, transmission in noisy channel, etc. Impulsive noise elimination is a common pre-processing step before feature image analysis. A gray scale image can be described by a function $f$, whose domain is a subset $F$, in the euclidean space $\mathbb{R}^{2}$ or the discrete space $\mathbb{Z}^{2}$, and its range is a subset $\mathcal{T}$ of $\overline{\mathbb{R}}=\mathbb{R} \cup\{-\infty, \infty\}$ or $\overline{\mathbb{Z}}=\mathbb{Z} \cup\{-\infty, \infty\}$. A noisy image of $f: F \subseteq \mathbb{Z}^{2} \rightarrow$ $\{0,1, \ldots, L\}$, where $L$ is the maximum value in the used intensity scale, is defined as an image $f_{r}: F \rightarrow\{0,1, \ldots, L\}$, affected by impulsive noise where a fraction $p \in[0,1]$ of the points in the domain of $f$, which are randomly selected with uniform probability and change its values with the following expression:

$$
f_{r}(x)=\tau(\rho(1) \leq p, \tau(\rho(1) \leq 0.5,0, L), f(x))
$$

Where $\tau\left(c, e_{v}, e_{f}\right)$ is the conditional function whose arguments are the logical condition to be evaluated, the expression to be evaluated $e_{v}$ when $\mathrm{c}=1$ (true) and the expression to evaluate $e_{f}$ for $c=0$ (false). In addition, $\rho(1)$ is a function 
that generates numbers with uniform distribution in the interval $(0,1)$. Note that the values to be assigned to function $f_{r}$ are the maximum and minimum in the gray scale.

In the literature impulsive noise removal methods have been purposed. One of the non-linear and robust filters most widely used is the standard median filter (SMF) [5], but as the noise level increases this filter tends to blur the image distorting its content. The Progressive Switched Median Filter (PMSF) [16], has the same drawbacks. The Adaptive Median Filter (AMF) [17], the Decision Based Algorithm (DBA) [13] and the Noise Adaptive Fuzzy Switching Median Filter for Salt-and-Pepper Noise Reduction (NAFSM) [8] are recent adaptive noise removal algorithms . Under the classical mathematical morphology framework opening and closing filters are often used for noise removal, however, even when such filters can give good results, as the level of noise degradation increases, these filters are not enough to recover good quality filtered images. Another image denoising filter commonly used is the median filter however it has the same disadvantages. In this work, an alternative impulsive noise removal filter is presented which brings satisfactory results even with a high degree of noise degradation. The proposed method preserves edges and fine feature details in the resulting images. Furthermore, experimental results shows that the proposed method has superior performance in noise removal than AMF and DBA methods when images are corrupted with high impulsive noise.

\section{Noise Removal with Classic Mathematical Morphology}

Classical mathematical morphology studies the images obtained after applying a number of operators which are the result of two basic operations composition, i.e., the mathematical morphology is a constructive theory [11]. These two elementary operations are defined.

Definition 1. The erosion and dilation of function $f: F \rightarrow \mathcal{T}$ by the structural function $b: B \rightarrow \mathcal{T}$, is defined as follows,

$$
\begin{aligned}
& \varepsilon_{b}(f)(x)=f \ominus b=\bigwedge_{y \in B}\{f(x+y)-b(y)\}, \\
& \delta_{b}(f)(x)=f \oplus b=\bigvee_{y \in B}\{f(x-y)+b(y)\} .
\end{aligned}
$$

The function $b$ given in the previous definition, is known as the structural function. In practice is common that the structural function $b$ is set up as the null function, in this case the erosion and dilation definitions are reduced to the following expressions,

$$
\begin{aligned}
& \varepsilon_{B}(f)(x)=f \ominus b=\bigwedge_{y \in B_{x}}\{f(y)\}, \\
& \delta_{B}(f)(x)=f \oplus b=\bigvee_{y \in \hat{B}_{x}}\{f(y)\} .
\end{aligned}
$$


Where $\hat{B}$ is the symmetric set of $B, \hat{B}=\{-x: x \in B\}$, and $B_{x}$ is the translation of $B$ to the point $x$. The set $B$ is called plane structural element. A fundamental feature of this structural element is that its size is lower than the image domain. Thus, the structural element acts as a local analyzer. Defined in this way the dilation and erosion are not the inverse the one of the other, and in general they are non invertible. This is our motivation to define other operations from their compositions.

Definition 2. The opening and closing of $f$ by $b$ are defined as follows.

$$
\gamma_{b}(f)=f \circ b=\delta_{b} \varepsilon_{b}(f) \quad \text { and } \quad \varphi_{b}(f)=f \bullet b=\varepsilon_{b} \delta_{b}(f)
$$

The derivative filters of the opening and closing operations composition are used in practice to eliminate noise in gray scale images. Figure 1 shows the result of applying these filters, using a flat structural element $B=\{(-1,0),(0,0),(0,1)$, $(1,0),(-1,0)\}$, to a contaminated image with impulsive noise under different levels of image degradation.

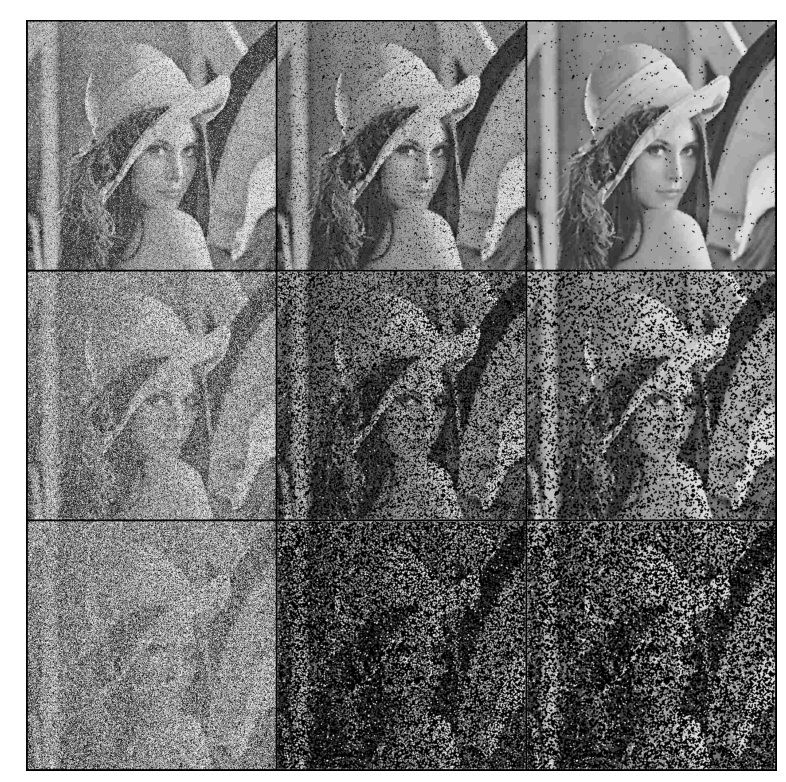

Fig. 1. From left to right, first row: An image with $20 \%$ of impulsive noise, opening morphological filter $\left(\gamma_{B}\right)$ and closing-opening $\left(\varphi_{B}\left(\gamma_{B}\right)\right)$, second row: image with impulsive noise of 50\%, morphological filter $\gamma_{B}$ and $\varphi_{B}\left(\gamma_{B}\right)$, third row: image with $70 \%$ of impulsive noise, morphological filter $\gamma_{B}$ and $\varphi_{B}\left(\gamma_{B}\right)$

As shown in Figure 1, the morphological filters open-close and close-open, are less effective as the noise degradation level increases in the image. In this paper 
two models adapted to noise that slightly modify the basic operations of erosion and dilation are presented, but turn out to have a very noticeable advantage over the original operators.

\section{Adaptive Mathematical Morphology}

As can be seen from the definitions (2) and (3), the classical mathematical morphology studies image transformations using as structural elements a small set of pixels of fixed size and form. There is a generalization of these operations with adaptive structural elements that depend on the spatial location of the image pixels or the image content, this area is called adaptive mathematical morphology $[6,7,9]$.

\subsection{Impulsive Noise Removal: Model 1}

A first approach to adapt the structural elements, is make them depend on its location in the space [1-4], then, once established the structural elements will remain fixed, i.e. they will not change with the input image. This approach is called "extrinsic". Let $f$ be a gray scaled image with domain in $F$, let us consider $\mathcal{A}$, a function that assigns to each point in the domain $F$, a set $\mathcal{A}(x) \subseteq F$, that can be different for every point. To define an adaptive structural element two parameters are considered, the first one is a criterion function $h$ that brings local information on the basis of local measurements as, brightness, contrast, curvature, thickness or orientation relative to the image $f$, in such a way, that the size and shape of the structural element is adapted to the local image characteristics. The second parameter is the tolerance with respect to the structural element in every point, thus, the set $V_{m}^{h}(x)$ is defined, containing all the points $y$ with $|h(y)-h(x)| \leq m$. In addition, it is needed that this set is connected by paths (path connected) with the usual topology in $F \subseteq \mathbb{R}^{2}$. This sets are known as Adaptive Neighborhood sets (AN sets). A fundamental feature of classical mathematical morphology is the adjuntion property of erosion and dilatation, since important algebraic properties of opening and closing operators depends on this characteristic [9]. In order to ensure that our operations meet such property, the structural element must be "auto-reflected", i.e. $\mathcal{A}(x)=\hat{\mathcal{A}}(x)=\{z \in F$ : $x \in \mathcal{A}(z)\}$, where, $x \in \mathcal{A}(y) \Leftrightarrow y \in A(x)$. The definition of erosion and dilation using adaptive structuring elements (auto-reflected AN set), are described as follows.

$$
\begin{aligned}
\epsilon_{\mathcal{A}}(f)(x) & =\bigwedge_{y \in \mathcal{A}(x)} f(y), \\
\delta_{\mathcal{A}}(f)(x) & =\bigvee_{y \in \mathcal{A}(x)} f(y) .
\end{aligned}
$$

Classic morphological filters do not satisfactorily recover the corrupted images with impulsive noise since this type of filters modifies all values in the image 
(even when the pixels have no noise) using the same structural element. Thus, in the first model we propose to modify the structural element according to their position in space, to modify only the necessary image information. Filtering follows the next scheme.

1. Establish a set $R$, of points containing all corrupted points with impulsive noise in the image.

2. Define two adaptive structural elements, one for the elements in the set $R$, and another one for the complement of $R, R^{c}$.

3. Apply adaptive opening and closing compositions, using these structural elements.

Let $f: F \subseteq \mathbb{Z}^{2} \rightarrow\{0,1,2, \ldots, L\}$, the gray scale image contaminated with impulsive noise. Note that points contaminated with impulsive noise in this image are the dots whose values are 0 or $L$. Let $R$ be the set containing all points where the noise function $f$ has the value 0 or $L$. We define the following sets:

$$
\mathcal{A}(x)=\left\{\begin{array}{c}
B_{x} \text { si } x \in R \\
\{x\} \text { si } x \in R^{c} .
\end{array}\right.
$$

Where $B$ is a fixed connex and symmetric structural element. Then, $\mathcal{A}(x)$ is an adaptive structural element. In figure 2, it is shown the result of applying the adaptive opening and closing filters to an image with varying degrees of degradation using the structural element $B=\{(-1,0),(0,0),(0,1),(1,0),(-1,0)\}$.

\subsection{Impulsive Noise Reduction: Model 2}

A priori limitations imposed on the size and shape of the structural element may not be the most suitable as other operations are applied, as a result of the elementary composition . A second approach called "intrinsic" [4,7,10], generalize the morphological operators, allowing the structural elements to depend on the input image. Definitions of erosion and dilation in this approach are similar to the definitions (2) and (3), with a slight but significant change in the structural elements. These definitions are described as follows.

$$
\begin{aligned}
& \epsilon_{\mathcal{A}^{f}}(f)(x)=\bigwedge_{y \in \mathcal{A}^{f}(x)} f(y), \\
& \delta_{\mathcal{A}^{f}}(f)(x)=\bigvee_{y \in \mathcal{A}^{f}(x)} f(y) .
\end{aligned}
$$

The set $A^{f}(x)$ is an adaptive structuring element, thus, $\mathcal{A}^{f}(x)$ is an auto-reflected set $\mathrm{AN}$. In this case, the restriction that the structural element is auto-reflected does not ensure the adjunction property, see [9]. The opening and closing opera-

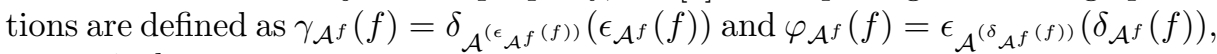
respectively. 


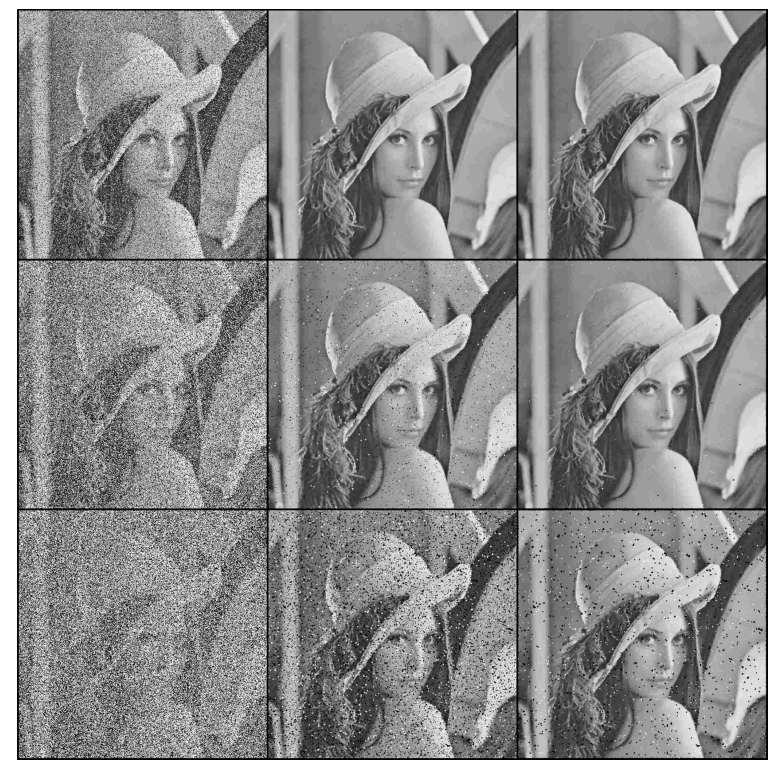

Fig. 2. From left to right, first row: An image with $20 \%$ of impulsive noise, opening morphological filtering $\left(\gamma_{\mathcal{A}}\right)$ and opening-closing $\left(\varphi_{\mathcal{A}} \gamma_{\mathcal{A}}\right)$, second row: image with $50 \%$ of impulsive noise, morphological filtering $\gamma_{\mathcal{A}}$ and $\varphi_{\mathcal{A}} \gamma_{\mathcal{A}}$, third row: image with $70 \%$ of impulsive noise, morphological filtering $\gamma_{\mathcal{A}}$ and $\varphi_{\mathcal{A}} \gamma_{\mathcal{A}}$

In this particular case, applying a first morphological operation to a noisy image we get a new image with less noise, we might consider to redefine the structural elements and streamline the process. To apply this second approach to eliminate impulsive noise, we propose the following scheme.

1. Establish a set $R$, of points containing all points corrupted with impulsive noise in the input image.

2. Define two adaptive structural elements, one for the elements in the set $R$, and another one for the complement of $R, R^{c}$.

3. Apply an erosion (dilation) according to these structural elements.

4. Define a new image, result of the previous step, as the input image and return to step 1 .

Let $f: F \subseteq \mathbb{Z}^{2} \rightarrow\{0,1,2, \ldots, L\}$, the gray scale image corrupted with impulsive noise and $R_{f}$ the set containing all points where the noise function $f$ has the value 0 or $L$. We define the adaptive structural elements as:

$$
\mathcal{A}^{f}(x)=\left\{\begin{array}{r}
B_{x} \text { if } x \in R_{f} \\
\{x\} \text { if } x \in R_{f}^{c}
\end{array}\right.
$$

Where $B$ is a fix connex and symmetric structural element. Figure 3 shows the result of applying the adaptive opening and closing filters to an image with 
varying degrees of degradation using structural element $B=\{(-1,0),(0,0),(0,1)$, $(1,0),(-1,0)\}$.

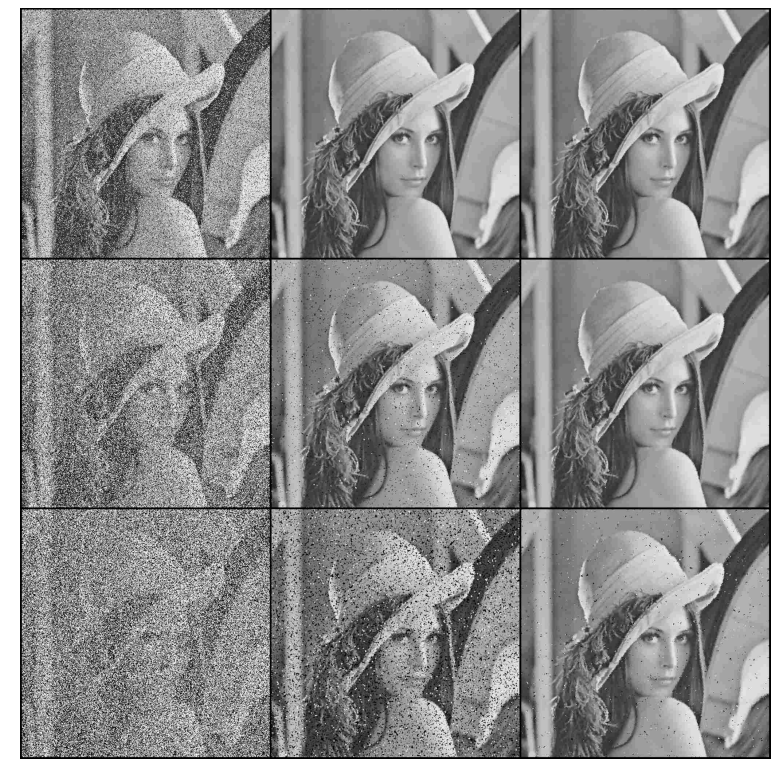

Fig. 3. From left to right, first row: image with $20 \%$ of impulsive noise, opening morphological filtering $\left(\gamma_{\mathcal{A}^{f}}\right)$ and opening-closing $\left(\varphi_{\gamma_{\mathcal{A}^{f}}}\left(\gamma_{\mathcal{A}^{f}}\right)\right)$, second row: image with $50 \%$ of impulsive noise, morphological filtering $\gamma_{\mathcal{A}^{f}}$ and $\varphi_{\gamma}\left(\gamma_{\mathcal{A}^{f}}\right)$, third row: image with $70 \%$ of impulsive noise, morphological filtering $\gamma_{\mathcal{A}^{f}}$ and $\varphi_{\gamma_{\mathcal{A}^{f}}}\left(\gamma_{\mathcal{A}^{f}}\right)$

\section{Comparison with Other Methods}

In this section the performance of the proposed method is evaluated and compared with other algorithms including SMF, PMSF, DBA, AMF y NAFSM using the Lenna image of size $510 \times 510$ degraded with different noise levels from $10 \%$ to $90 \%$ with $10 \%$ increments. The image restoration quality is measured with two metrics: Peak signal-to-noise ratio, PSNR, a metric widely used for image quality evaluation [14] and SSIM [15], a metric based on the fact that the object structures are independent of the lighting conditions. The evaluation results are presented in Table 1, and Figure 5 shows the performance of the methods for each metric. From the results we observed that for noise level lower than $30 \%$ the NAFSM, PMSF, AMF and DBA algorithms have slightly better results than the proposed methods under the used metrics, however, when noise corruption is higher that $40 \%$, the results of the proposed algorithm are better than the PMSF, AMF and DBA algorithms, however, when the proposed 
method is compared against the NAFSM algorithm, it can be noticed that the NAFSM algorithm has better performance when PSNR metric is used while our algorithm obtains better results under the SSIM metric. Figure 6 shows the resulting images from the application of the algorithms SMF, DBA, AMF, NAFSM and the proposed adaptive algorithm when images are corrupted with impulsive noise of $70 \%, 80 \%$ and $90 \%$. In this figure it can be seen however, when the proposed method is compared against the NAFSM algorithm, it can be noticed that the NAFSM algorithm has better performance when PSNR metric is used while our algorithm obtains better results under the SSIM metric and that the proposed algorithm preserves better image details, edges and light than the other the DBA, AMF algorithms. Finally, in tables 2 and 3 the SMF, PMSF, DBA, AMF, NAFSM, and the proposed method are compared using the Barbara and Einstain 4 images both of size $510 \times 510$ pixels with impulsive nose in the range of $10 \%$ to $90 \%$, here it can be observed that the proposed method is better than the PMSF, AMF, and DBA algorithms and has similar performance to the NAFSM algorithm.

In practice it was observed that the proposed algorithm converges with different number of iterations of adaptive filters. Using the structural element $B=\{(-1,0),(0,0),(0,1),(1,0),(-1,0)\}$, for a noise level of $10 \%$ it is enough the application of an opening operator; for noise level of $20 \%$ and $30 \%$ it must be applied an opening followed by a closing; for a noise level of $40 \%, 50 \%$ and $60 \%$ it must be applied the composition of filters openingclosingopening; for noise level between $70 \%$ and $80 \%$ the filter composition must be closing-opening-closing opening; while for noise level of $90 \%$ the composition filter must be opening -closing-closing-opening. It was also noted that the application of more filters to the restored images does not diminish their quality.

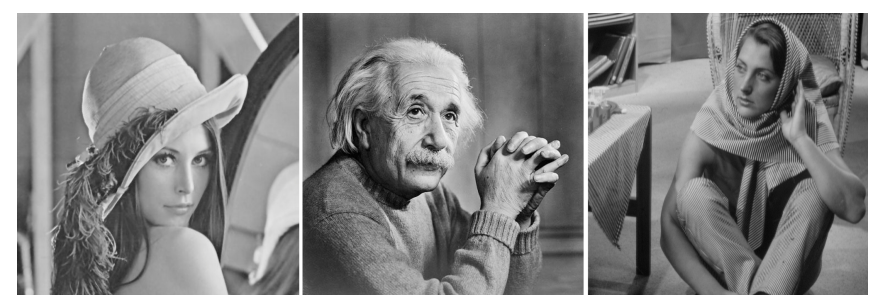

Fig. 4. From left to right: Lenna, Einstein and Barbara

\section{Results}

In figure 1, it can be notice that classical morphological filters are quite effective in removing noise, when noise is not as aggressive but, once the degree of degradation increases, these filters are ineffective and slow. Figure 2 shows that 
Impulsive Noise Removal by Adaptive Mathematical Morphology

Table 1. Values of PSNR in $\mathrm{dB}$ and SSIM for different algorithms in the Lenna image contaminated with different levels of impulsive noise

\begin{tabular}{|c|c|c|c|c|c|c|c|c|c|c|c|c|} 
Noise & \multicolumn{2}{|c|}{ SMF } & \multicolumn{2}{c|}{ PSMF } & \multicolumn{2}{c|}{ DBA } & \multicolumn{2}{c|}{ AMF } & \multicolumn{2}{c|}{ NAFSM } & \multicolumn{2}{c|}{ Proposed } \\
$\%$ & PSNR & SSIM & PSNR & SSIM & PSNR & SSIM & PSNR & SSIM & PSNR & SSIM & PSNR & SSIM \\
\cline { 2 - 12 } 10 & 35.86 & 0.9757 & 37.01 & 0.9903 & 43.82 & 0.9991 & 41.64 & 0.9981 & 40.80 & 0.9979 & 38.24 & 0.9971 \\
20 & 29.73 & 0.9260 & 32.23 & 0.9790 & 39.49 & 0.9971 & 38.36 & 0.9961 & 37.41 & 0.9947 & 35.54 & 0.9944 \\
30 & 23.80 & 0.7566 & 28.39 & 0.9485 & 36.24 & 0.9936 & 35.83 & 0.9929 & 35.52 & 0.9906 & 34.11 & 0.9918 \\
40 & 19.12 & 0.4879 & 24.88 & 0.8647 & 34.09 & 0.9873 & 33.77 & 0.9877 & 34.04 & 0.9849 & 33.02 & 0.9895 \\
50 & 15.28 & 0.2330 & 15.14 & 0.3113 & 31.35 & 0.9745 & 31.71 & 0.9789 & 32.57 & 0.9765 & 31.88 & 0.9854 \\
60 & 12.37 & 0.1040 & 12.27 & 0.1800 & 29.05 & 0.9546 & 30.05 & 0.9672 & 31.40 & 0.9672 & 30.76 & 0.9797 \\
70 & 9.99 & 0.0939 & 9.93 & 0.0997 & 26.20 & 0.9128 & 27.96 & 0.9450 & 30.20 & 0.9544 & 29.55 & 0.9700 \\
80 & 8.18 & 0.0533 & 8.14 & 0.0571 & 23.68 & 0.8461 & 25.95 & 0.9068 & 28.78 & 0.9337 & 28.30 & 0.9523 \\
90 & 6.68 & 0.0249 & 6.67 & 0.0268 & 20.13 & 0.6762 & 22.95 & 0.8118 & 26.43 & 0.8847 & 26.07 & 0.9030 \\
\hline
\end{tabular}
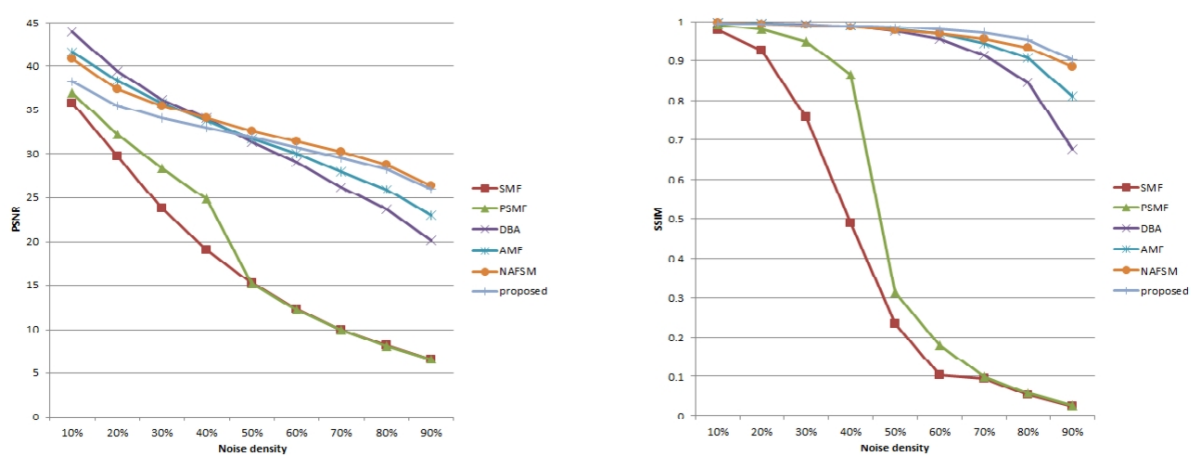

Fig. 5. Comparative graphs of PSNR in DB and SSIM for noise removal algorithms in Lenna image contaminated with different levels of impulsive noise

there is an important improvement after noise removing in the corrupted image, however the execution time of the algorithm is slow when properly parameter settings are able to obtain a satisfactory restoration. An important parameter is the size of the structural element, the noise is removed faster if a bigger structural element is used, however the image tends to get blurred loosing definition in the edges, in real applications the size of the structural element must be set according to the size of the features that must remain in the input image. Another reason is that the modified points are always the same. Suppose that we have 3 points, $x, y$ and $z$, horizontal aligned in the discrete domain of the image with values in the set $\{0,1, \ldots, L\}$ and, $f(x)=0$ y $f(y)=f(z)=L$. Then, after applying an erosion with a structural element that fulfill these three points, then, the value of the function in $y$ changes its value to $L$, and let us suppose that the point $z$ is no longer noise, i.e. its value is neither 0 nor $L$. Let us suppose that the following operation it must be an erosion, then, since we consider again the point $z$ to be filtered, this point remain a noisy point with value of zero. Thus, 
Marisol Mares Javier, Carlos Guillén Galván, Rafael Lemuz López

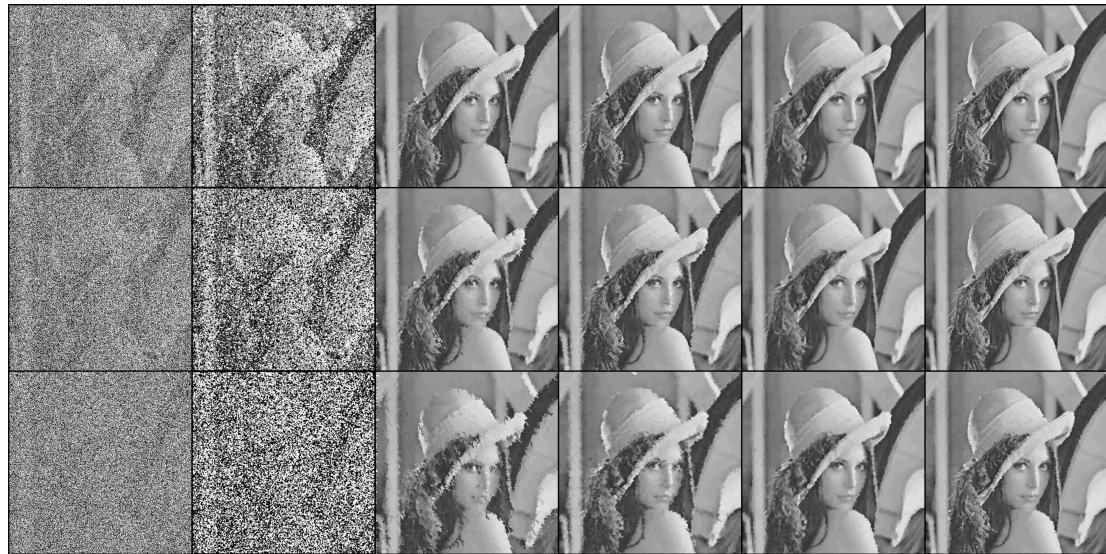

Fig. 6. Results of noise removal algorithms for the Lenna image. From left to right: 1st column: image with impulsive noise of $70 \%, 80 \%$ and $90 \%$, 2nd column: Output of MF, 3rd column: DBA, 4th column: AMF,5th column: NAFSM, 6th column: the proposed method

Table 2. Values of PSNR in $\mathrm{dB}$ and SSIM for different algorithms in the Einstein image contaminated with different levels of impulsive noise

\begin{tabular}{|c|c|c|c|c|c|c|c|c|c|c|c|c|c|} 
Noise & \multicolumn{2}{|c|}{ SMF } & \multicolumn{2}{c}{ PSMF } & \multicolumn{2}{c|}{ DBA } & \multicolumn{2}{c|}{ AMF } & \multicolumn{2}{c|}{ NAFSM } & \multicolumn{2}{c|}{ Proposed } \\
$\%$ & PSNR & SSIM & PSNR & SSIM & PSNR & SSIM & PSNR & SSIM & PSNR & SSIM & PSNR & SSIM \\
\cline { 2 - 13 } 50 & 14.94 & 0.3020 & 20.23 & 0.6610 & 27.06 & 0.9287 & 26.59 & 0.9257 & 27.90 & 0.9371 & 26.45 & 0.9356 \\
60 & 12.08 & 0.1699 & 12.03 & 0.1797 & 25.30 & 0.8892 & 23.34 & 0.8953 & 26.92 & 0.9134 & 25.56 & 0.9153 \\
70 & 9.77 & 0.0961 & 9.72 & 0.1022 & 23.62 & 0.8342 & 24.10 & 0.8581 & 26.02 & 0.8854 & 24.71 & 0.8881 \\
80 & 7.91 & 0.0551 & 7.88 & 0.0586 & 21.57 & 0.7492 & 22.61 & 0.7987 & 24.96 & 0.8478 & 23.68 & 0.8449 \\
90 & 6.43 & 0.0265 & 6.41 & 0.028 & 19.16 & 0.6084 & 20.82 & 0.7049 & 23.44 & 0.7818 & 22.29 & 0.7758 \\
\hline
\end{tabular}

the third approach shows that the elimination of noise streamlines every step if, now begin to discard these points that need no longer to be filtered.

\section{Conclusions}

We proposed two models of adaptive mathematical morphology for impulsive noise removal that have advantages over the classical mathematical morphology model. The results are positive in both models, as they can properly adapted to the essential characteristics of impulsive noise. The model based on the "intrinsic" approach turns out to have advantages over "extrinsic" approach, since it is adjusted in every step to the new intensities of the image to be filtered. As future work, we propose to use other structural elements to improve the results and correct the edge distortion in the images. In addition, the complexity analysis between algorithms could be evaluated. 
Table 3. Values of PSNR in $\mathrm{dB}$ and SSIM for different algorithms in the Barbara image contaminated with different levels of impulsive noise

\begin{tabular}{|c|c|c|c|c|c|c|c|c|c|c|c|c|} 
Noise & \multicolumn{2}{|c|}{ SMF } & \multicolumn{2}{c|}{ PSMF } & \multicolumn{2}{c|}{ DBA } & \multicolumn{2}{c|}{ AMF } & \multicolumn{2}{c|}{ NAFSM } & \multicolumn{2}{c|}{ Proposed } \\
$\%$ & PSNR & SSIM & PSNR & SSIM & PSNR & SSIM & PSNR & SSIM & PSNR & SSIM & PSNR & SSIM \\
\cline { 2 - 9 } 50 & 14.67 & 0.3012 & 19.38 & 0.603 & 24.78 & 0.8844 & 24.21 & 0.8777 & 25.99 & 0.9112 & 24.44 & 0.8977 \\
60 & 11.89 & 0.1693 & 15.70 & 0.4070 & 23.44 & 0.8395 & 23.17 & 0.8429 & 25.09 & 0.8832 & 23.57 & 0.8715 \\
70 & 9.66 & 0.0912 & 9.63 & 0.0978 & 22.19 & 0.7840 & 22.15 & 0.8050 & 24.19 & 0.8484 & 22.78 & 0.8380 \\
80 & 7.90 & 0.0496 & 7.88 & 0.0527 & 20.60 & 0.6918 & 21.14 & 0.7471 & 23.21 & 0.8048 & 21.80 & 0.7899 \\
90 & 6.43 & 0.0266 & 6.42 & 0.0278 & 18.15 & 0.5215 & 19.54 & 0.6431 & 21.88 & 0.7291 & 20.72 & 0.7164 \\
\hline
\end{tabular}

\section{Acknowledgments}

We thank to CONACyT, PRODEP and VIEP-BUAP for the financial support. Also, we thank the reviewers for their contributions to improve this research and writting of the manuscript.

\section{References}

1. Angulo, J.: Morphological Bilateral Filtering and Spatially-Variant Adaptive Structuring Functions. In: Soille, P., Pesaresi, M., Ouzounis, G.K. (eds.) ISMM 2011. LNCS, vol. 6671, pp. 212-223. Springer, Heidelberg (2011)

2. Bouaynaya, N., Schonfeld, D.: Spatially variant morphological image processing: theory and applications. In: Proceedings of SPIE, vol. 6077, pp. 673-684 (2006).

3. Bouaynaya, N., Schonfeld, D.: Theoretical foundations of spatially-variant mathematical morphology part II: Gray-level images. IEEE Transactions on Pattern Analysis and Machine Intelligence 30(5), 837-850 (2008)

4. Debayle, J., Pinoli, J.: Spatially Adaptive Morphological Image Filtering using Intrinsic Structuring Elements. Image Analysis and Stereology 24(3), 145-158 (2005)

5. Huang, T., Yang G., and Tang G.: A fast two-dimensional median filtering algorithm. IEEE Trans. Acoust., Speech, Signal Processing 27(1), 13-18 (1979)

6. Heijmans H.J., and Ronse C.: The Algebraic Basis of Mathematical Morphology I, Dilations and Erosions. Comput. Vision Graphics Image Processing 50(3), 245-295 (1990)

7. Maragos, P.A., Vachier, C.: Overview of adaptive morphology: Trends and perspectives. In: Proc. of IEEE International Conference on Image Processing, pp. 2241-2244 (2009)

8. Vin Toh, K.K. and Mat Isa, N.A.: Noise Adaptive Fuzzy Switching Median Filter for Salt-and-Pepper Noise Reduction. IEEE Signal Processing Letters 17(3), 281-284 (2010)

9. Roerdink, J.B.T.M.: Adaptivity and group invariance in mathematical morphology. In: 16th IEEE International Conference on Image Processing (ICIP), pp. 2253-2256 (2009)

10. Salembier, P.: Study on nonlocal morphology operators. In: 16th IEEE International Conference on Image Processing (ICIP), pp. 2269-2272 (2009)

11. Serra, J.: Image Analysis and Mathematical Morphology. Academic Press, London, United Kingdom, (1982) 
Marisol Mares Javier, Carlos Guillén Galván, Rafael Lemuz López

12. Soille, P.: Morphological Image Analysis: Principles and Applications, 2nd Ed., Springer-Verlag, Berlin Heidelberg, Germany, (2003)

13. Srinivasan, K.S., and Ebenezer, D.: A New Fast and Efficient Decision-Based Algorithm for Removal of High-Density Impulse Noises. IEEE Signal Processing Letter 14(3), 189-192 (2007)

14. Wang, Z., Bovik, A.C.: Mean squared error: love it or leave it? A new look at signal fidelity measures. IEEE Signal Processing Magazine 26(1), 98-117 (2009)

15. Wang Z., Bovik, A.C., Sheikh H.R., and Simoncelli, E.P.: Image quality assessment: From error visibility to structural similarity. IEEE Transactions on Image Processing 13(4), 600-612 (2004)

16. Wang, Z. and Zhang, D.: Progressive Switching Median Filter for the Removal of Impulse Noise from Highly Corrupted Images. IEEE Trans. on Cir. and Sys 46(1), 78-80 (1999)

17. Zhao, Y., Li D., Li Z.: Performance enhancement and analysis of an adaptive median filter. In: International Conference on Communications and Networking, pp. 651-653, (2007) 